\title{
A Rare Case of "Large Twisted Dermoid Cyst" in an 82-year-old Lady with Diverticulitis and Septicemia: A Case Report
}

\author{
Manjula BC
}

\begin{abstract}
Dermoid cyst is a germ cell tumor. Eighty percent occurs during reproductive years. One to two percent undergoes malignant transformation. This is a case of a large dermoid cyst ovary measuring $10 \times 12 \mathrm{~cm}$ in an 82-year-old lady who complained of acute on chronic pain abdomen associated with constipation. CT scan revealed a heterogeneous mass replacing ovary. Emergency laparoscopy done revealed a large collection of pus in peritoneal cavity filling POD and extending up to the subhepatic region with massively distended bowel loops. The right ovary was torted 3.5 times. To the best of our knowledge, this is the only case described in the literature of torsion ovary in a lady above the age of 80 years with diverticulitis managed laparoscopically.
\end{abstract}

Keywords: Diverticulitis, Septicemia, Twisted dermoid cyst.

How to cite this article: Manjula BC. A Rare Case of "Large Twisted Dermoid Cyst" in an 82-year-old Lady with Diverticulitis and Septicemia: A Case Report. J South Asian Feder Menopause Soc 2018;6(2):132-134.

Source of support: Nil

Conflict of interest: None

Date of received: 10 January 2018

Date of acceptance: 27 January 2018

Date of publication: July 2018

\section{INTRODUCTION}

Germ cell tumors of the ovary are derived from totipotent germ cells of the ovary. Dermoid cyst or a mature cystic teratoma is the commonest type of ovarian germ cell tumors. These are the most common benign ovarian tumors with a reported prevalence of up to $20 \% .{ }^{1}$ They are bilateral in 10 to $15 \%$ of cases. Most dermoid cysts are diagnosed in women of reproductive age and are often discovered incidentally in asymptomatic women. Complications associated with dermoid cyst include

\section{Fellow}

Department of Gynae Endoscopy, Pushpawathi Singhania Research Institute, South Delhi, India

Corresponding Author: Manjula BC, Fellow, Department of Gynae Endoscopy, Pushpawathi Singhania Research Institute, South Delhi, India, e-mail: manjulabiju0@gmail.com torsion, rupture, and infection though these are rare events.

\section{CASE REPORT}

An 82-year-old postmenopausal lady p212 presented with lower abdominal pain and fever associated with nausea over chronic constipation. She was first seen by gastroenterologist owing to the age and clinical presentation.

\section{On Examination}

Pulse: Tachycardia

BP: $110 / 70 \mathrm{~mm} \mathrm{Hg}$.

\section{Febrile}

$P / A$ : Tenderness right iliac fossa

Blood routine: Total and differential count raised

Blood culture: The growth of S. fecium.

Was diagnosed to have diverticulitis with septicemia and was treated for the same. But she had no much relief.

Computed tomography abdomen revealed markedly dilated sigmoid colon with edematous serosal layer indicating infection (Fig. 1).

A heterogeneous $10 \times 12$ mass in right iliac fossa seen indicating a dermoid cyst (Fig. 2).

A diagnosis of the twisted dermoid cyst was made and taken up for emergency laparoscopic cystectomy.

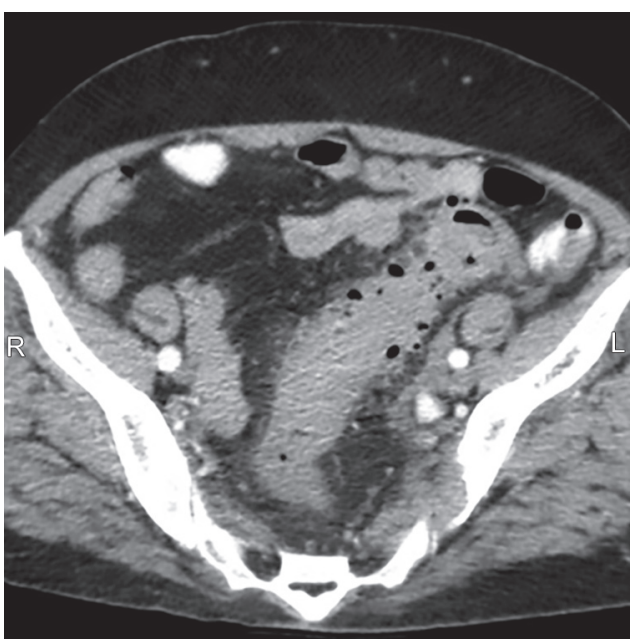

Fig 1: Marked dilatation of sigmoid colon with thickened borders indicating edema of the serosal wall 


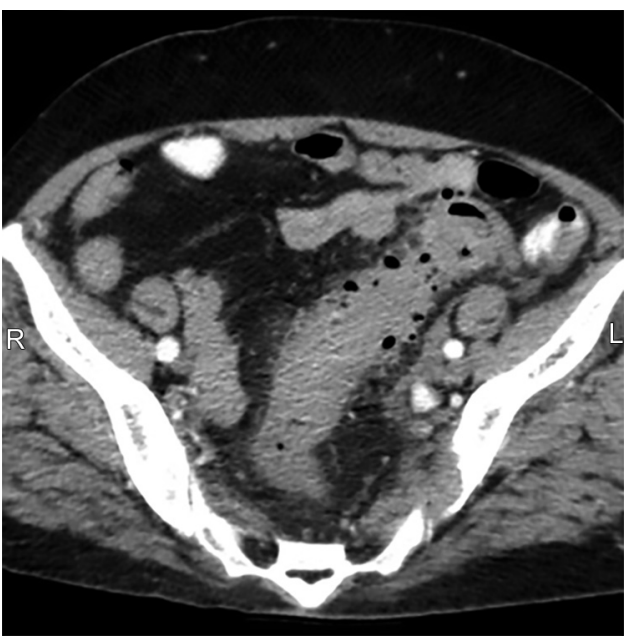

Fig 2: Heterogeneous mass on right side of pelvic cavity indicating dermoid cyst

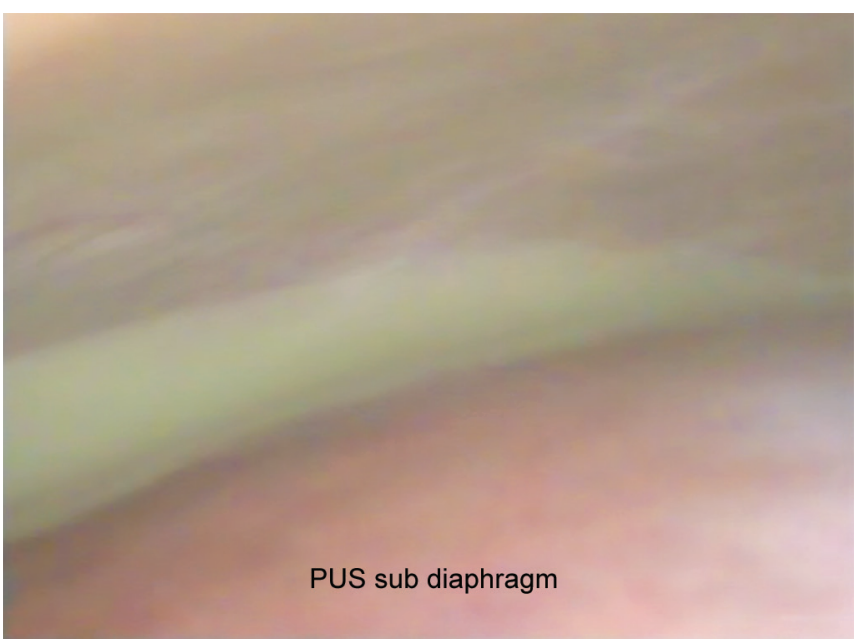

Fig 4: Pus extending up to subdiaphragmatic region

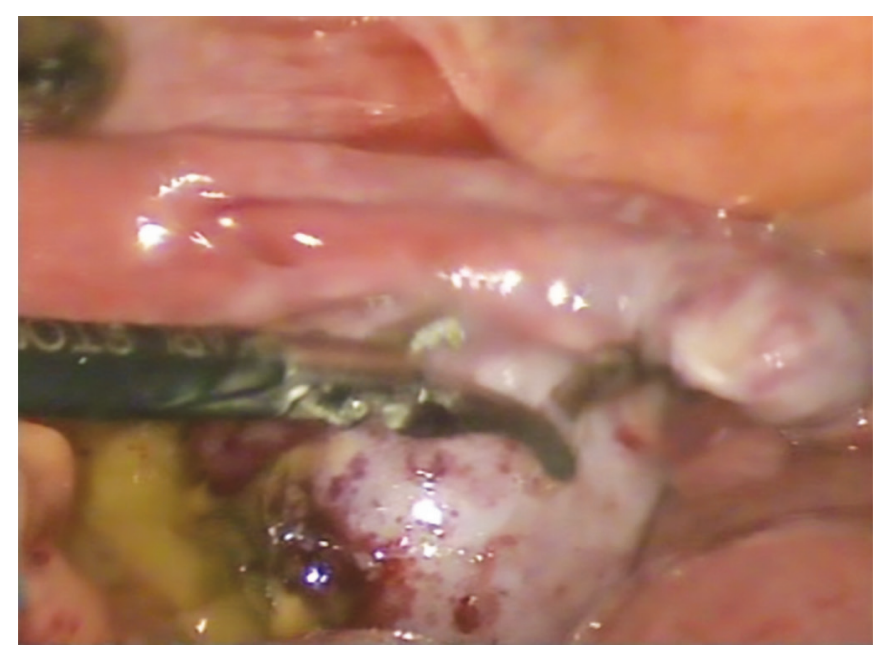

Fig 6: Cheesy material from the cyst

On opening up, there was a huge collection of pus extending from POD with markedly distended bowel loops (Fig. 3) to subdiaphragmatic region (Fig. 4). All this made surgery very difficult. The cyst was twisted clockwise 3.5 times (Fig. 5), untwisted, cauterized and cut. Cyst invariably ruptured during the procedure as it invariably

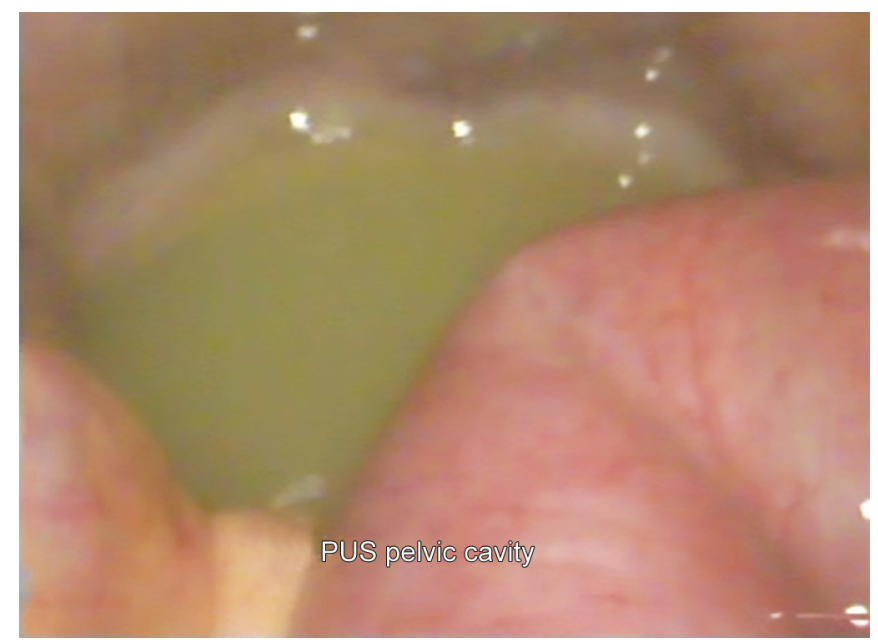

Fig 3: Pus in POD with markedly distended bowel loops

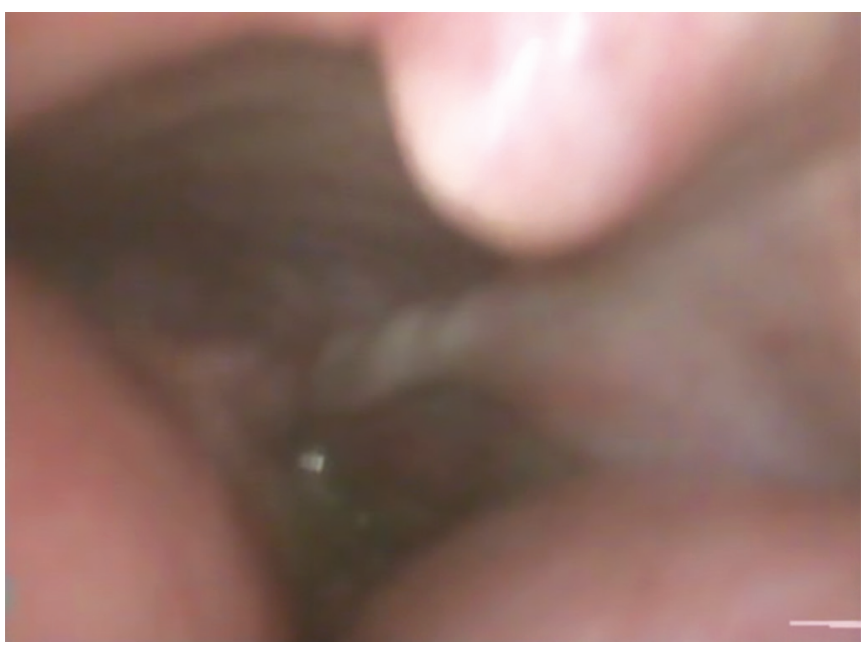

Fig 5: Torsion of the cyst pedicle

occurs during laparoscopy showing cheesy material (Fig. 6) indicating a dermoid cyst. The specimen was sent for histopathological examination.

Histopathology report showed necrosis of dermoid cyst confirming twisting of the cyst.

\section{DISCUSSION}

Dermoid cyst arises from the germ cells arrested after the first meiotic division. Dermoid cyst constitutes about $95 \%$ of benign cystic teratoma. Its incidence is about $15-20 \%$ amongst ovarian tumors. The tumor is bilateral in about 15-20\%. Dermoid cysts occur most commonly in the reproductive age group. Unlike other germ cell tumors of the ovary, dermoids have a wider age distribution and may be encountered from infancy to old age. ${ }^{2}$ The risk of malignancy is age-related and is greater in postmenopausal women. ${ }^{3}$ They are the most common ovarian neoplasm found in adolescence and during pregnancy. Mature cystic teratoma grows at an average rate of $1.8 \mathrm{~mm}$ each year. The chance of malignancy is about $1-2 \%$. 
There are many reported cases of ovarian tumor in postmenopausal women, but dermoid cyst is very rare. The oldest age group in which dermoid cyst found in the literature is 72 years reported in a scientific journal of Hamadan University of Medical Sciences 2009 vol 16, number (sn 53) page 49-51. We present to you this case because of its uniqueness and age at which it presented that is at the age of 82 years.

A dermoid cyst is a relatively common tumor that is an infrequent cause of abdominal and flank pain. ${ }^{4}$ Pain is often related to the size pain when it occurs, is from mass effect and ovarian torsion. Torsion is the most common 15 to $20 \%$, and rupture is an uncommon $1 \%$ complication. Partial or complete twisting of ovarian suspensory ligament causes severe pain, nausea and tissue necrosis. ${ }^{5}$ In our case, the patient had presented with fever, nausea, abdominal pain and chronic constipation. Blood culture revealed the growth of $S$. fecium as a result of which a diagnosis of diverticulitis with septicemia was made. But the patient had no relief from pain in spite of antibiotics and analgesics though there was a regression of fever. As $\mathrm{CT}$ abdomen revealed a heterogeneous mass in the pelvic cavity on the right side a diagnosis of the dermoid cyst with the possibility of being twisted was made. Acute severe abdominal pain may indicate torsion or rupture. Owing to their high-fat content, dermoid cysts tend to be more buoyant in the pelvis than other types of neoplasms. Therefore they are more likely to result in torsion.

These tumors have a classic radiographic appearance. The incidence of malignant elements in a teratoma is low (approximately 1-2\%). Gonadal dermoid cysts occur mostly during the reproductive years, between the age of 20-40 years. They are frequently multicystic and contain sebaceous fluid as well as hair, teeth, bone, and skin. Typically, these tumors contain mature tissue of ectodermal (skin, brain), mesodermal (muscle, fat) and ectodermal (mucinous or ciliated epithelium) origin. ${ }^{4}$ They have characteristic CT scan appearance with fat fluid level attenuation and calcification or ossification, as demonstrated in Figure 2 of the mass and ovarian torsion is common. It is mostly composed of ectodermal tissue. which gives them their characteristic features of sebaceous glands, sweat glands, hair, and teeth.

Measurement of CA 125 levels can be helpful for distinguishing between benign and malignant adnexal masses, especially in postmenopausal women. In our case, CA 125 was within normal limits. Tumor markers especially CA 125 and CA 19-9 have a special predictive value in women with dermoid cyst. ${ }^{6}$ The patient was taken up for emergency laparoscopy once we got the report. We had great difficulty in operating on this patient because of marked distension of bowel loops as a result of sigmoiditis. There was a huge collection of pus in peritoneal cavity POD extending up to the subdiaphragmatic region. Ovarian torsion was seen on laparoscopy. Her pain was most probably due to the ischemic complication of the enlarged ovary twisting around its pedicle. Upon gross dissection the tumor demonstrated a classic appearance of a mature cystic teratoma, demonstrating endodermal, mesodermal tissues. The patient underwent rightsalpingo-oophorectomy and through the peritoneal wash after the pus was retrieved for culture and sensitivity examination. The patient had an uncomplicated postoperative course.

\section{CONCLUSION}

Though dermoid cyst is commonest in reproductive age group $15 \%$ incidence has been reported in posmenopausal age group. Be vigilant in diagnosing a case of acute abdomen in women irrespective of age group as concomitantly two pathologies can, and the patient should be examined in toto and adnexal torsion is always a surgical emergency

\section{REFERENCES}

1. Hoo WL, Yazbek J, et al. Expectant management of ultrasonically diagnosed ovarian dermoid cysts: is it possible to predict outcome?. Ultrasound in Obstetrics and Gynecology 2010;36(2):235-240.

2. Gupta AK, Madan R, et al. Sonographic spectrum of ovarian dermoid. J Obstet Gynecol India 2005;55:170-173.

3. Jitsumori M, Munakata S, et al. Malignant Transformation of Mature Cystic Teratoma Diagnosed after a 10-Year Interval. Case reports in obstetrics and gynecology. 2017;2017:90-92.

4. Williams JB, Orr SC. An unusual presentation of a right ovarian dermoid cyst. J Emerg Med 2011;41(3):296-297.

5. Kakani A, Bannerjee I, et al. A Huge Dermoid Cyst in Postmenopausal Women with Third Degree Uterine Prolapse. J South Asian Feder Menopause Soc 2013;1(1):43-44.

6. Ozturk N, Sok FC. Laparoscopic management of ovarian dermoid cysts: a review of 47 cases. Ann Saudi Med 2004;24(5):357-360. 